\title{
Radio evidence for binary super massive black holes
}

\author{
R. D. Ekers \\ CSIRO Astronomy and Space Vimera and Pembroke Roads, Marsfield, NSW 2122, Australia \\ email: ron.ekers@csiro.au
}

\begin{abstract}
I present examples of radio AGN with binary nuclei which provide the direct radio evidence for binary Super Massive Black Holes (SMBH) driving the AGN activity. There is also other evidence for distorted radio morphology and periodic variability which may indicate the presence of a second (inactive) SMBH. Finally I enumerate a number of possible radio tracers for the binary SMBH merger events.
\end{abstract}

Keywords. Super Massive Black Holes (SMBH), binary, AGN

\section{Introduction}

The previous talk (Kellermann 2015) has summarized the evidence associating Super Massive Black Holes (SMBH) with AGN activity. When the AGN is radio loud we have an excellent SMBH tracer observable throughout the universe and VLBI observations have sufficient angular resolution to separate close (pc scale) SMBHs and to observe changes in the jet direction. All methods to investigate binary SMBHs need a radio loud AGN on at least one SMBH component to provide a tracer visible at radio wavelengths.

Radio images need to have sufficient resolution and quality to distinguish multiple AGN from multiple hot spots in a single AGN or to identify multiple images of a gravitational lensed AGN.

\section{Multiple AGN}

Spatially resolved binary AGN. 3C75, in Abel 400, (Owen et al. 1985) is one of the best examples of a binary AGN with two clearly separated AGN each with a nuclear source and very similar classical radio galaxy jet and lobe structure (Fig. 1). The separation of the two nuclei in $3 \mathrm{C} 75$ is $7.6 \mathrm{kpc}$. There are now many other examples of other similar double AGN e.g. PKS2359-139, 3C442, PKS2149-15, 3C338. In all cases the nuclei are separated by tens of kpc. Resolution at radio wavelengths is easily sufficient to see multiple AGN at smaller separation but these are much rarer. $0402+379$ is the most compact known binary AGN with two compact radio components separated by $7 \mathrm{pc}$ (Rodriguez et al. 2006).

On much smaller scales we can ask whether the evolving core-jet structures seen in the multi-epoch VLBI observations, described by Kellermann (2015) in the previous talk, would be affected by a second SMBH. For example, in 3C279, described by Homan et al. (2003), we see an abrupt change in jet direction at 3 milliarcseconds from the nucleus (1 kpc deprojected). There is a tendency for component trajectories to follow "pre-existing channels" and we see components deflected at this point in 1998 and again in 2004 could this be a deflection by a second SMBH?

Other evidence for multiple AGN. Another option which can now be explored uses the new wideband spectra-polarimetry capabilities in JVLA (Perley et al. 2011) and ATCA 


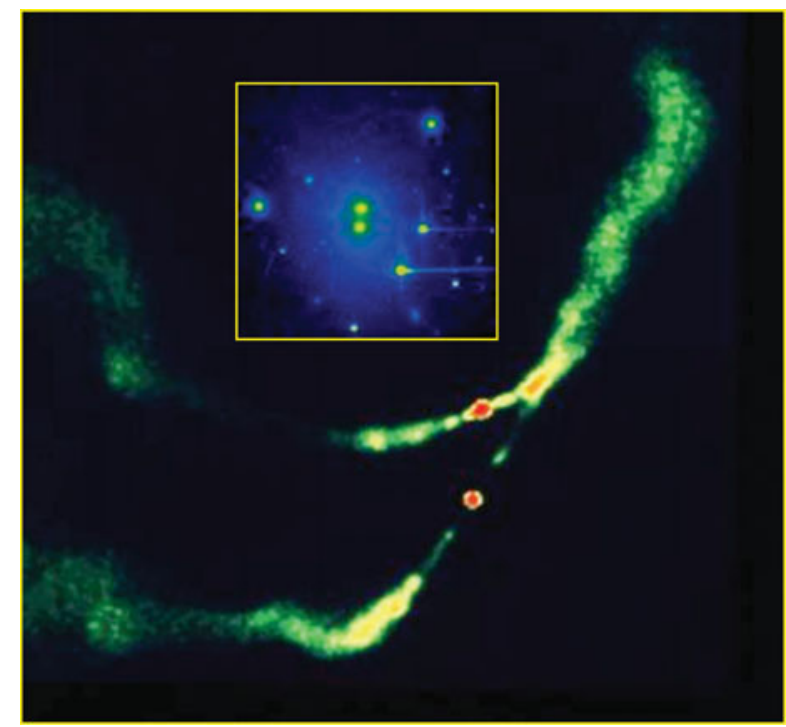

Figure 1. VLA observations of the radio galaxy 3C75, inset optical double nuclei

(Wilson et al. 2011) is to search for double rotation measures. It is expected that the line of sight to the two nuclei traverse regions of different density or magnetic field, and this will produce a double peak in the rotation measure synthesis even if the individual components are not spatially resolved.

Small diameter radio sources scintillate as a consequence of the propagation of the radio waves through irregularities in the interstellar medium. This is an analogue of the optical twinkling of stars due to irregularities in the troposphere. If a radio source scintillates this could be used to find double AGN. The sub pc scale of interest is easily accessible by looking for multiple peaks in scintillation structure function at sub-day time scales.

Periodic flux variability provides indirect evidence for the presence of a second SMBH. For example, OJ287 has shown quasi periodic variability with a 12 year cycle which was modelled by Valtonen et al. (2008) as a precessing binary black hole. Further observations, including observations of the changes in the resolved jet structure (Valtonen \& Pihajoki 2013), have not directly confirmed initial predictions but have resulted in more complex models involving black hole spin and helical jets.

\section{A systematic radio census of binary SMBH}

Burke-Spolaor (2011) used archival Very Long Baseline Interferometry (VLBI) data for 3114 radio loud AGN, to search for binary supermassive black holes on pc scales. The only example found, was the 7 pc binary AGN, 0402+379, already mentioned.

Probability of detecting a binary $S M B H$. Note that the probability of detecting radio emission from an AGN is only $1 \%$ at Jy flux density levels, hence the probability of detecting both components in a binary SMBH is only $0.01 \%$. At lower (mJy) flux levels this probability could increase to $10 \%$, hence a $1 \%$ probability for two active SMBHs. Future studies will need much larger samples to find the close SMBH binaries. This requires more sensitivity to go to fainter sources but, sadly, SKA1 no longer has the long baseline needed to do this so we will have to wait for SKA2. A further complication which arises is the possible effect of one AGN on a second nearby SMBH. This could either quench the second AGN by blowing its accretion material away or enhance activity by 

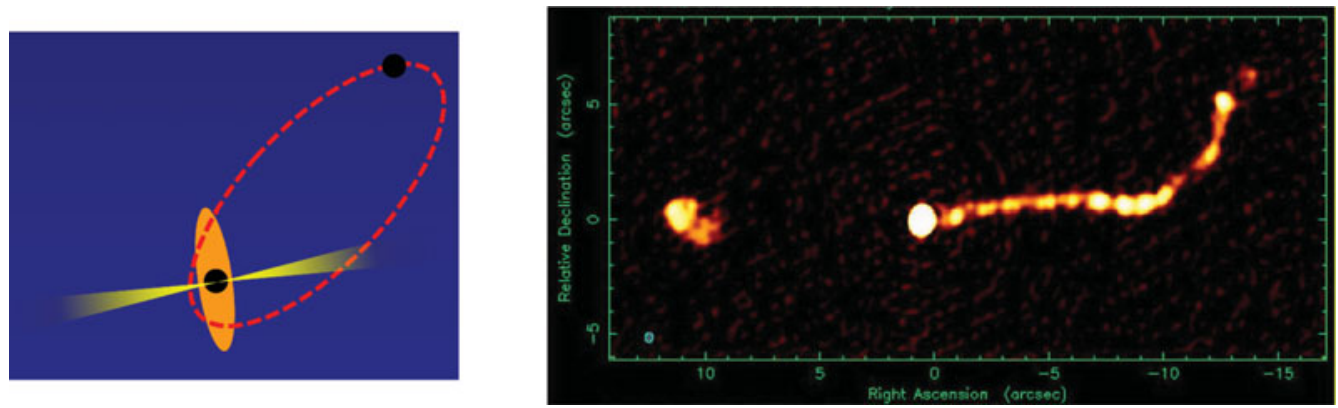

Figure 2. ATCA observations of radio galaxy 0637-752 with binary SMBH model

disturbing its accretion disk and increasing the amount of material accreted. The most likely outcome is that any collimated jet of flux from the first AGN will miss the second AGN and have little effect.

Binary scale sizes. AGN are good for probing the high-mass end of the SMBH distribution: $>10^{8} \mathrm{M}_{\odot}$. The inspiral time scale due to gravitational radiation is $10^{8}$ years (1\% of age of universe) while the AGN phase lasts $10^{9}$ years i.e. about 10 times longer. The dynamical stalling radius where the well known dynamical effects may stop working is about $3 \mathrm{pc}$ and this is just the range of angular scale sizes accessible to VLBI techniques. Unfortunately, with earth scale baselines it is hard to reach the $<0.2 \mathrm{pc}$ scale sizes for which gravitational radiation is detectable by pulsar timing. The Burke-Spolaor (2011) analysis suggests that binary pair evolution of supermassive black holes (masses $>10^{8} \mathrm{M}_{\odot}$ ) spend less than $500 \mathrm{Myr}$ in progression from the merging of galactic stellar cores to within the purported stalling radius for supermassive black hole pairs. The data show no evidence for an excess of stalled binary systems at small separations.

Triple SMBH System! Deane et al. (2014) have recently published a claim for a triple SMBH. This is SDSS J1502+1115, a $z=0.39$ QSO with double peaked OIII. It contains one dust obscured AGN with a double flat spectrum radio core, separation $138 \mathrm{pc}$, and this is claimed to be a double SMBH. There is a second unobscured AGN with a third compact radio component $7.4 \mathrm{kpc}$ away from the double core. Wrobel, Walker, \& Fu (2014) obtained higher quality VLA data which indicates that the obscured AGN is actually a double sided AGN with very compact lobes and a single central SMBH. This source then becomes another example of a large separation binary SMBH.

\section{Dynamical effects}

Binary modulation of AGN activity. This spectacular "chain of beads" AGN, PKS 0637-752 (Fig. 2) could be explained by a bound second SMBH periodically plunging through the disk of the active SMBH which maintains its spin axis but has a burst of activity as the accretion disk is perturbed on each plunge, (Godfrey et al. 2012). The bend $\left(12^{\prime \prime}\right) 80 \mathrm{kpc}$ to the west may be an axis re-orientation event?

Astrometric measurement of orbit: 3C66. Sudou et al. (2003) suggested that 3C66B was in a 1 year orbit, based on astrometric VLBI measurements. This has been disproved by Jenet et al. (2004) because the gravitational wave emission would have already been detectable in the pulsar timing data. 


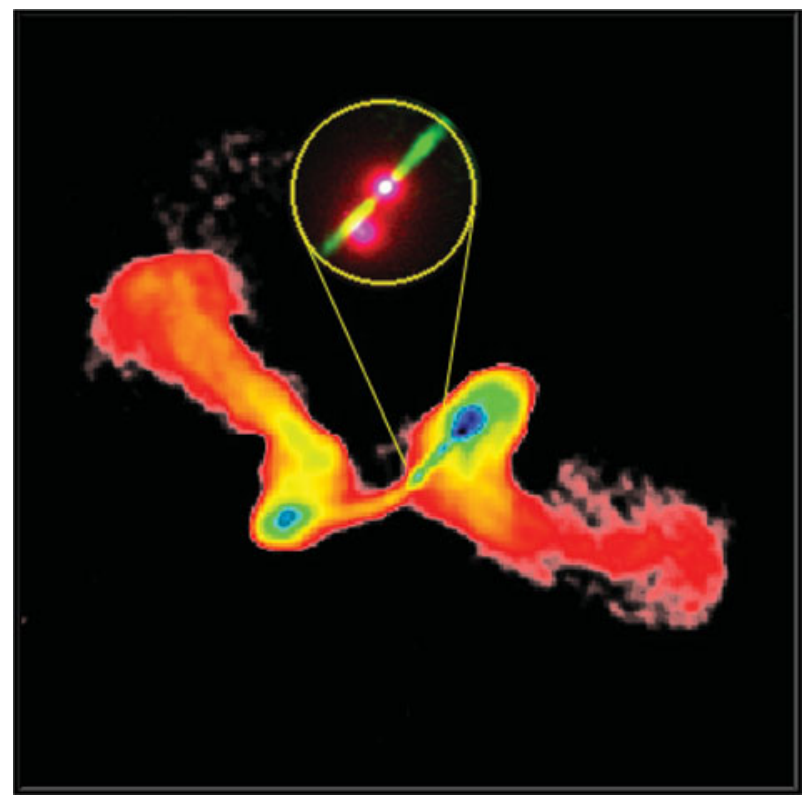

Figure 3. VLA observations of NGC326

\section{Alignment of radio axes}

In a giant radio galaxy, such as NGC 6251, the axis direction has been maintained for the life of the source, i.e. $>10^{9}$ years. This is the normal situation seen in radio galaxies and this extraordinary long term stability of the jet axis is strong evidence that jet orientations are regulated by black hole spins (e.g. Begelman et al. 1984). More rarely we see examples where there is a change in axis direction. These are rare but about a dozen good examples are now known. They are referred to as the X sources. NGC326 (Ekers et al. 1978, Murgia et al. 2001) is the poster child example of an X-shaped radio galaxy in which the jet axis changed direction (Fig. 3). Rees (1978) summarised a range of possible models for changing direction of the spin axis and Merritt \& Ekers (2002) explored the specific implications of merging binary SMBHs. These X structures occur in a few \% of all radio galaxies but there is no consensus on whether this is an axis change due to a binary SMBH merger or whether it is due to a hydrodynamical effect in the external medium (e.g. Saripalli et al. 2013). Another proposed alternative is that the X-shaped sources may result from two pairs of jets that are associated with a pair of unresolved AGNs tracing a binary SMBH that have not yet merged (Lal \& Rao 2007).

\section{Observable consequences of SMBH mergers}

In addition to the direct evidence for the presence of binary SMBH there are a number of potentially observable consequences of a binary SMBH merger.

- Spin axis changes generate X sources (see previous section)

- Dynamical kicks during the merger process will generate misaligned core jet structures.

- AGN is displaced from the centre of the galaxy.

- AGN activity is disrupted by the kick and emission is quenched.

- One sided jets may be a consequence of relative velocity or density asymmetries resulting from a merger event. 
- Electromagnetic pulses may be generated in the surrounding plasma by the intense gravity wave generated by the merger.

Any future detection of gravitational waves (by LIGO, LISA etc.) from SMBH mergers or other gravitational events will need to be identified with their host galaxies to measure redshift but such identification will need more accurate positions than the many degree error boxes possible with the gravitational wave detectors. Any simultaneous transient radio emission could be used to obtain a sufficiently accurate position to make an identification. The wide field of view and possible rapid follow up of the future radio instruments will provide these opportunities.

\section{Summary}

Radio continuum emission can identify double AGNs and hence binary SMBH. There are many examples of binary activity on scales of a few kpc separation but these are very rare at pc separation. We have some evidence for periodic modulation of the radio properties which could be caused by a binary SMBH. The morphology of the X shaped radio sources shows some good examples of possible jet re-orientation or precession but interpretation of these observations is not unambiguous.

\section{References}

Begelman, M. C., Blandford, R. D., \& Rees, M. J. 1984, Rev. Mod. Phys., 56, 255

Burke-Spolaor, S. 2011, MNRAS, 410, 2113-2122

Deane, R. P., Paragi, Z., Jarvis, M. J., Coriat, M., Bernardi, G., Fender, R. P., Frey, S., Heywood, I., Klckner, H.-R., Grainge, K., \& Rumsey, C. 2014, Nature, 511, 5760

Ekers, R. D., Fanti, R., Lari, C., \& Parma, P. 1978, Nature, 276, 588-590

Godfrey, L. E. H.., Lovell, J. E. J.., Burke-Spolaor, S., Ekers, R. D., Bicknell, G. V., Birkinshaw, M., Worrall, D. M., Jauncey, D. L., Schwartz, D. A., Marshall, H. L., Gelbord, J., Perlman, E. S., \& Georganopoulos, M. 2012, ApJL, 758, L27

Homan, D. C., Lister, M. L., Kellermann, K. I., Cohen, M. H., Ros, E., Zensus, J. A., Kadler, M.,\& Vermeulen, R. C. 2003, ApJL, 589, L9-L12

Jenet, F. A., Lommen, A., Larson, S. L., \& Wen, L. 2004, ApJ, 606 799-803

Kellermann, K. I. 2015, IAU Symposium 312, Star Clusters and Black Holes in Galaxies and Across Cosmic Time, ed. Fukun Liu

Lal, D. V. \& Rao, A. P. 2007, MNRAS, 374, 1085

Merritt, D. \& Ekers, R. D. 2002, Science, 297, 1310-1313

Murgia, M., Parma, P., De Ruiter, H. R., Bondi, M., Ekers, R. D., \& Fanti, R., . Fomalont, E. B. 2001, $A \mathscr{E} A, 380,102-116$

Owen, F. N., O’Dea, C. P., Inoue, M., \& Eilek, J. A. 1985, ApJL, 294, L85-L88

Perley, R. A., Chandler, C. J., Butler, B. J., \& Wrobel, J. M. 2011, ApJL, 739, L1

Rees, M. J. 1978, Nature, 275, 516

Rodriguez, C., Taylor, G. B., Zavala, R. T., Peck, A. V., Pollock, L. K., \& Romani, R. W. 2006, ApJ, 646, 49

Saripalli, L., Malarecki, J. M., Subrahmanyan, R., Jones, D. H., \& Staveley-Smith, L. 2013, MNRAS, 436, 690-696

Sudou, H., Iguchi, S., Murata Y., \& Taniguchi, Y. 2003, Science, 300, 1263-1265

Valtonen, M., Kidger, M., Lehto, H., \& Poyner, G. 2008, Astronomy \& Astrophysics, 477, 407-412

Valtonen, M. \& Pihajoki, P. 2013, A\& A, 557, 28-32

Wilson, W.E., Ferris, R.H., Axtens, P., Brown, A., Davis E., Hampson, G., Leach, M., Roberts, P., Saunders, S. Koribalski, B.S., \& 23 coauthors 2011, MNRAS, 416, 832-56

Wrobel, J. M., Walker, R. C., \& Fu, J. H. 2014, ApJL, 792, L8 